\section{$\varepsilon_{8}$}

${ }^{1}$ Respiratory Medicine, Royal Children's Hospital, Melbourne, Australia

${ }^{2}$ Department of Paediatrics, The University of Melbourne, Victoria, Australia

${ }^{3}$ Murdoch Children's Research Institute, Victoria, Australia

${ }^{4}$ Anatomical Pathology, Royal Children's Hospital, Melbourne, Australia

${ }^{5}$ Paediatric Surgery, Royal Children's Hospital, Melbourne, Australia

Correspondence to Dr Shivanthan Shanthikumar, Respiratory Medicine, Royal Children's Hospital, 50 Flemington Road, Parkville Melbourne, VIC 3052, Australia; shivanthan.shanthikumar@rch. org.au

Received 23 December 2016 Revised 5 March 2017 Accepted 7 March 2017

Published Online First 3 April 2017

\title{
A toddler with a cough and wheeze refractory to treatment
}

\author{
Shivanthan Shanthikumar, ${ }^{1,2,3}$ Tommy Lwin, ${ }^{1}$ Chung W Chow, ${ }^{2,4}$ Joe Crameri, ${ }^{5}$ \\ Jo Harrison $1,2,3$
}

\section{TOMMY LWIN (MEDICAL STUDENT)}

A 20-month-old girl weighing $12 \mathrm{~kg}$ presented with 2 weeks of worsening dry cough and wheeze. Aside from respiratory symptoms she was otherwise well, without fever or weight loss. There was no history of foreign body inhalation or choking. She had no significant personal or family medical history, and her immunisations were up to date. Prior to admission she had been treated for 1 week for a community-acquired pneumonia with oral amoxicillin and roxithromycin, as well as for a first presentation asthma exacerbation with oral prednisolone $(2 \mathrm{mg} / \mathrm{kg})$ and inhaled salbutamol, three hourly. Despite these interventions no clinical improvement had been noted. On examination, her respiratory rate and oxygen saturations were normal. She had mild respiratory distress, a loud biphasic wheeze and a prolonged expiratory phase. A chest X-ray (CXR) demonstrated hyperinflation of the right lung (figure 1A). Given the suspicion of a foreign body obstructing a right-sided airway, a rigid bronchoscopy was performed which showed an isolated submucosal lesion in the right main bronchus causing airway narrowing (figure 1B). To further characterise the lesion, a CT of the chest was performed, which demonstrated a subcarinal mass (figure 1C), causing significant extrinsic compression of the distal right main bronchus and bronchus intermedius. There was no intraluminal perforation of the mass.

\section{SHIVANTHAN SHANTHIKUMAR (RESPIRATORY FELLOW) AND JO HARRISON (RESPIRATORY CONSULTANT)}

The most likely differential diagnoses for a subcarinal mass in this patient include a neoplastic lesion (lymphoma, or germ cell tumour), an infectious mass (tuberculous (TB) or non-TB mycobacterium (NTM)). There are no associated features to help determine whether a malignancy or infection is more likely; however, there has been no exposure to $\mathrm{TB}$, making a TB infection less likely. Another potential diagnosis which would be very uncommon in this age group is an inflammatory mass due to sarcoidosis. To make a diagnosis, a tissue sample is needed, and traditionally this would be obtained via either open surgery or video-assisted thoracoscopic surgery. These procedures are invasive with a prolonged recovery time and potentially serious side effects. ${ }^{1}$ In adult medicine, less invasive methods of obtaining a biopsy have become routine, and these techniques are beginning to be used in the paediatric population (see table 1).

The use of endobronchial ultrasound-guided transbronchial needle aspiration (EBUS TBNA) in a paediatric patient was first described in 2009 , and since then it has been used across many centres. Gilbert et $a l^{2}$ reviewed 21 paediatric patients who underwent EBUS TBNA using a specially designed bronchoscope equipped with a distal ultrasound probe allowing real-time ultrasound imaging at the time of biopsy (performed via operating channel). No significant adverse events were reported. Dhooria et al ${ }^{1}$ reviewed 67 paediatric patients who had either an EBUS TBNA performed, or the same equipment was placed in the oesophagus to obtain a transoesophageal biopsy (EUS-B-FNA). There was no difference in diagnostic yield between EBUS TBNA and EUS-B-FNA with a calculated diagnostic sensitivity of $79.1 \%$ for EBUS TBNA or EUSB-FNA. One issue with EBUS TBNA identified in adults is the relatively high false-negative rate of needle aspirates in diagnosing lymphomas with prominent fibrosis and a mixed cellular population. ${ }^{2}$ This limitation is important to take into account in paediatric practice, given the higher incidence of lymphoma.

Another technique that could have been considered in this case is cryotherapy in which a cryoprobe is passed to the desired area via a bronchoscope. Cryotherapy has multiple uses in adult pulmonology including removal of foreign bodies and transbronchial biopsy to diagnose granulomatous disease, infection, interstitial lung disease, lung cancer and post-transplant rejection. Its use in the paediatric population is more limited; however, Zhang $e t \mathrm{al}^{3}$ recently reported a series of 12 patients who had cryotherapy to remove a foreign body. In 2 of the 12 cases, cryotherapy damaged the airway mucosa causing asymptomatic partial obstruction of the airway.

Transbronchial biopsies performed via the operating channel of a bronchoscope without image guidance have also been used in the diagnosis of paediatric subcarinal masses with a series of 28 paediatric patients demonstrating that it is a safe procedure. ${ }^{4}$ When compared with EBUS TBNA, a limitation of this methodology is the lack of image guidance, although an advantage is that it can be performed via a smaller bronchoscope.

After a multidisciplinary discussion including an adult respiratory physician specialising in EBUS, a paediatric general surgeon and an anatomical pathologist, it was decided that although EBUS TBNA would be feasible, a needle aspirate might not provide a sample sufficient for diagnosis if the mass was due to a lymphoma such as Hodgkin's lymphoma which has a non-uniform population of tumour cells, and hence may be missed on an aspirate. Hence, the patient was referred for a thoracoscopic biopsy. 

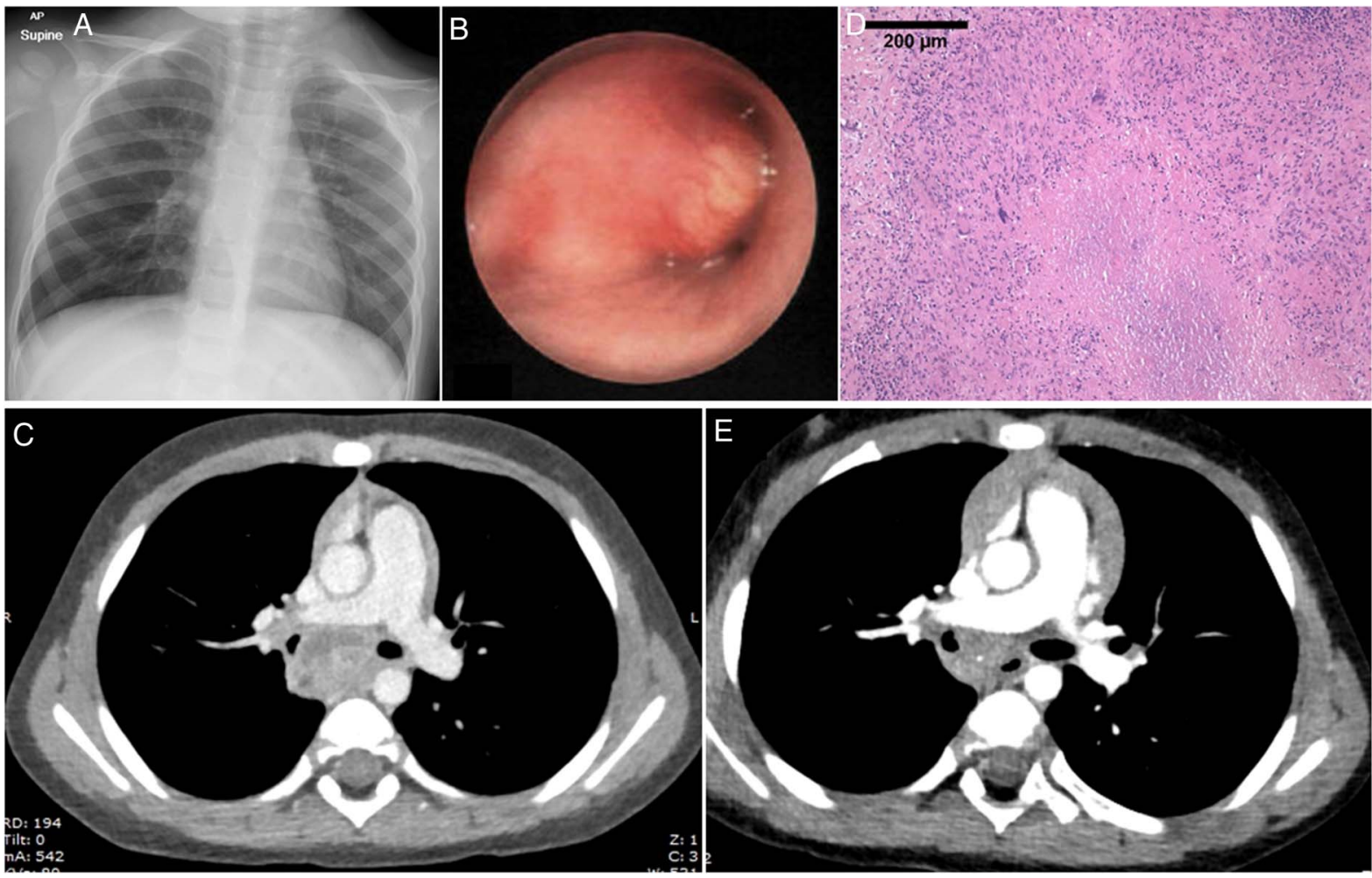

Figure 1 (A) Chest X-ray at presentation showing hyperinflation of right lung. (B) Image from rigid bronchoscopy showing occlusion of right main bronchus by submucosal lesion. (C) Initial CT scan showing subcarinal mass compressing the right main bronchus. (D) Lymph node biopsy demonstrating necrosis with nuclear debris surrounded by a thin layer of caseation and epithelioid cells with occasional Langhans giant cells. (E) Repeat CT scan showing reduction in size of mass with ongoing right main bronchus compression.

TL

While planning for a biopsy, other investigations were performed to help identify the cause of the mass. A full blood examination including blood film showed no signs of inflammation or malignancy. Tumour markers ( $\alpha$ fetoprotein and $\beta$ hCG) to investigate a germ cell tumour were normal. A bronchial lavage showed no acid-fast bacilli on Ziehl-Neelsen stain, no malignant cells, and mycobacterial cultures were negative. To further investigate TB, a tuberculin skin test (TST) was performed and was positive at $20 \mathrm{~mm}$ of induration. An Interferon Gamma Release Assay (QuantiFERON-TB Gold) (IGRA) for TB was negative, and early morning gastric aspirates were not done.

\section{JOE CRAMERI (PAEDIATRIC SURGEON)}

Thoracoscopy revealed a large lymph node wrapped around the vagus nerve and azygous vein with no discernible tissue plane. It was not suitable for excision. An incisional biopsy was sent for frozen section analysis which suggested a mycobacterial infection. A biopsy of nodal tissue was obtained, and pus was aspirated from the node and sent for analysis.

\section{SS, JH AND CW CHOW (ANATOMICAL PATHOLOGIST)}

Given the strongly positive TST and the frozen section findings, the likely cause of the patient's subcarinal mass is a mycobacterial infection. A TST of $20 \mathrm{~mm}$ raises a high suspicion of TB; however, NTM infection can also cause a positive TST. The lymph node biopsy showed many granulomas with focal stellate necrosis and a thin layer of caseation surrounded by regularly palisaded epithelioid cells with occasional Langerhans-type giant cells (see figure 1D). These features are strongly suggestive of a mycobacterial infection, although the exact nature of the infective organism could not be determined with certainty. In order

Table 1 Summary of less invasive bronchoscopic biopsy techniques for paediatric patients

\begin{tabular}{|c|c|c|c|c|c|c|}
\hline Study & Technique & Scope size (channel size) & Route & $\begin{array}{l}\text { Patients (youngest } \\
\text { patient) }\end{array}$ & $\begin{array}{l}\text { Adequate } \\
\text { material }\end{array}$ & $\begin{array}{l}\text { Diagnostic } \\
\text { sample }\end{array}$ \\
\hline Gilbert et $a l^{2}$ & EBUS TBNA & $6.9 \mathrm{~mm}$ & LMA & 21 (18 months, 10 kg) & $95 \%$ & $47 \%$ \\
\hline Dhooria et al ${ }^{1}$ & $\begin{array}{l}\text { EBUS TBNA or } \\
\text { EUS-B-FNA }\end{array}$ & $6.9 \mathrm{~mm}$ & LMA & 67 (3 years) & $92 \%$ & $56.9 \%$ \\
\hline Zhang et $a l^{3}$ & Cryotherapy* & $\begin{array}{l}4.8 \mathrm{~mm}(2.2 \mathrm{~mm}) \text { or } 4.0 \mathrm{~mm} \\
(2.0 \mathrm{~mm})\end{array}$ & $\begin{array}{l}\text { Mouth or } \\
\text { LMA }\end{array}$ & 12 (10 months) & NA & NA \\
\hline $\begin{array}{l}\text { Goussard } \\
\text { et } a l^{4}\end{array}$ & Transbronchial biopsy & $4.0 \mathrm{~mm}(2.0 \mathrm{~mm})$ & LMA & 28 (9 months) & & $57 \%$ \\
\hline
\end{tabular}

${ }^{*}$ No biopsy samples taken.

EBUS TBNA, endobronchial ultrasound-guided transbronchial needle aspiration; EUS-B-FNA, transoesophageal biopsy. 
to determine whether it is a TB or non-TB infection, the tissue sample was sent for culture and PCR testing for both TB and NTM. As there was some delay until the results of culture and PCR were available, consideration was given to empiric TB treatment. A delay in obtaining these results is not unique to our institution, and in this situation some guidelines recommend TB treatment. However, as the patient remained systemically well, had a negative IGRA and there was no history of contact with TB infection, a decision was made not to start treatment. Another potential tool for discriminating between NTM and TB infection would be the use of dual skin testing with a traditional TST and a Mycobacterium avium sensitin; however, the authors are unaware of a Mycobacterium avium sensitin that is licensed for clinical use in humans and widely commercially available, and it is not possible at our hospital.

\section{TL}

The results of mycobacterial culture were negative; however, using PCR, mycobacterial DNA was detected, and following Sanger sequencing the DNA was found to originate from Mycobacterium avium. Treatment with azithromycin $(10 \mathrm{mg} / \mathrm{kg}$ once time per day), Rifampicin ( $10 \mathrm{mg} / \mathrm{kg}$ once time per day) and ethambutol $(15 \mathrm{mg} / \mathrm{kg}$ daily $)$ was commenced. A repeat CXR prior to starting treatment was normal. A screen for immune deficiencies (FBE, lymphocyte subsets, immunoglobulins, tetanus and pneumococcal antibodies) was also normal, although testing for an interferon- $\gamma$ or IL-12 deficiency was not performed.

\section{SS AND JH}

Intrathoracic disease is an extremely uncommon presentation of NTM, with cervical lymphadenitis being the most common site of infection. There are multiple case reports of NTM causing a similar presentation to our patient, and based on these reports we would expect our patient to have a good outcome. Nolt et $a l^{5}$ identified 43 cases of reported intrathoracic NTM disease between 1930 and 2003. In this group, 40\% of patients had a similar presentation to our patient with respiratory symptoms in isolation, while the remainder also had constitutional symptoms including fever, weight loss and night sweats. Of note, the average TST measurement was $7 \mathrm{~mm}$. In the group of patients with isolated respiratory symptoms, the median duration of treatment was 6 months, although the details of treatment were not described. Only $3 / 43$ patients suffered relapse of their disease. Fergie $e t a l^{6}$ reviewed eight cases of intrathoracic NTM infection, seven of which were in immunocompetent patients and one patient with acquired hypogammoglobulinaemia. In the seven immunocompetent patients, all were cured, after they received a variety of treatments involving two to three antibiotics used for 2-14 months. Some cases also had either partial or complete surgical resection. Aside from one case, the patients in both series were thought to be immunocompetent, although it is unclear how thoroughly they were assessed for immune deficiency, and hence it is still reasonable to investigate patients with this unusual presentation. Of note, the median age of presentation in both case series was 24 months, which is similar age to that of our patient.

Based on the described cases and guidelines for treatment of NTM disease, a prolonged course of antibiotics will be needed.
A repeat CT scan (figure 1D) 7 months into treatment showed significant reduction in the size of the subcarinal nodal mass; however, there was ongoing right main bronchus compression. Clinically she had noisy breathing on exertion but no exercise limitation or systemic symptoms. Twelve months of treatment will be completed with repeat imaging at that stage to assess treatment response.

\section{CONCLUSION}

This case of intrathoracic NTM disease presenting with wheeze unresponsive to asthma treatment highlights some important lessons for health professionals looking after paediatric patients:

1. Wheeze that does not respond to bronchodilator therapy warrants further investigation for airway obstruction.

2. Based on experience in adult patients, new techniques for obtaining biopsies via a flexible bronchoscopy are being used in paediatric patients, and when planning a procedure, clinicians should always try and choose the least invasive method of attaining an adequate sample. At this stage, there is no clear 'gold standard' method, and the choice will be based on patient factors (age, size), pathology factors (mass location, mass size and potential diagnoses) and clinician factors (personal experience, equipment available, support from colleagues such as adult respiratory physicians). It is important to note that these novel modalities may not result in a diagnostic sample, and patients may need to proceed to an open biopsy.

3. This case highlights that some patients with NTM infections can have strongly positive TST result. Therefore, in TST-positive cases, without risk factors for TB, who are clinically stable, withholding TB treatment while awaiting microbiological confirmation of the causative organism can be considered.

4. Intrathoracic NTM cases have been reported and are thought to occur in mainly immunocompetent patients who generally have good outcomes with prolonged courses of combination antibiotic therapy.

Contributors SS and JH contributed to the conception and planning of the article and act as joint guarantors of the content. All authors contributed to the writing and review of the article.

Competing interests None declared.

\section{Patient consent Obtained.}

Provenance and peer review Not commissioned; externally peer reviewed.

\section{REFERENCES}

1 Dhooria S, Madan K, Pattabhiraman V, et al. A multicenter study on the utility and safety of EBUS-TBNA and EUS-B-FNA in children. Pediatr Pulmonol 2016;51:1031-9.

2 Gilbert CR, Chen A, Akulian JA, et al. The use of convex probe endobronchial ultrasound-guided transbronchial needle aspiration in a pediatric population: a multicenter study. Pediatr Pulmonol 2014;49:807-15.

3 Zhang L, Yin Y, Zhang J, et al. Removal of foreign bodies in children's airways using flexible bronchoscopic CO2 cryotherapy. Pediatr Pulmonol 2016;51:943-9.

4 Goussard P, Gie RP, Kling S, et al. The diagnostic value and safety of transbronchial needle aspiration biopsy in children with mediastinal lymphadenopathy. Pediatr Pulmonol 2010;45:1173-9.

5 Nolt D, Michaels MG, Wald ER. Intrathoracic disease from nontuberculous mycobacteria in children: two cases and a review of the literature. Pediatrics 2003;112:e434.

6 Fergie JE, Milligan TW, Henderson BM, et al. Intrathoracic Mycobacterium avium complex infection in immunocompetent children: case report and review. Clin Infect Dis 1997;24:250-3. 